\title{
Significance and fate of septal fibrosis of the liver
}

\author{
Márcia Maria de Souza, Miguel Tolentino Jr., \\ Bárbara C.A. Assis, Ana Cristina de Oliveira Gonzalez, \\ Tânia Maria Correia Silva, Zilton A. Andrade* \\ Laboratory of Experimental Pathology, Gonçalo Moniz Research Center-FIOCRUZ_Rua Valdemar Falcão 121, \\ 40295-001 Salvador, BA, Brazil
}

Received 15 September 2005; received in revised form 24 January 2006; accepted 30 January 2006

Available online 24 March 2006

\begin{abstract}
Septal fibrosis commonly occurs during chronic diseases of the liver. It is experimentally reproduced in a proportion of rats treated with pig-serum, and in $100 \%$ of rats infected with Capillaria hepatica. These models have only been used in relatively short-term studies. To contribute to the natural history and significance of hepatic septal fibrosis it is important to disclose its fate after prolonged observation, and following partial or total withdrawal of its inciting cause.

Adult Wistar rats were sacrificed 3, 6, 9 and 12 months following inoculation of 800 embryonated eggs of $C$. hepatica. Besides routine histology, liver sections were submitted to immunohistochemical, immunofluorescence and ultrastructural techniques for the identification of cells and extracellular matrix components present in the fibrous septa. Septal blood vessels were studied after portal vein perfusion with India-ink, while the hepatic functional profile and levels of anti-C. hepatica antibodies were determined in collected sera.

Results revealed that all parasites were already dead 2 months from inoculation, and the accumulated eggs in the liver lost their capacity to embryonate around the 4th-6th month, when progressive reduction in the number of cells and in the amount of collagen occurred in the septa.

Septal fibrosis persisted throughout the time of experimentation (12 months). This fibrosis was seen as a supporting stroma for septal vessels that conducted portal blood directly to the sinusoids. Thus, persistence of fibrosis was probably related to its morphological and functional association with blood vessels
\end{abstract}

(C) 2006 Elsevier Ireland Ltd. All rights reserved.

Keywords: Capillaria hepatica; Septal hepatic fibrosis; Fibrosis reversibility

\section{Introduction}

A peculiar type of septal fibrosis of the liver regularly occurs in Capillaria hepatica-infected rats [1]. During its life cycle, this helminth dies out inside the liver, around the 20th day of infection, soon after sexual maturity and egg lying. Necro-inflammatory lesions are then formed around the disintegrating parasites and their eggs. When such focal parasitic lesions start exhibiting signs of encapsulation and progressive resorption, which usually takes place around the 25th-30th day following inoculation, thin and long fibrous septa begin

\footnotetext{
* Corresponding author. Tel.: +55 71 31762206; fax: +55 7131762297.

E-mail address: zilton@ cpqgm.fiocruz.br (Z.A. Andrade).
}

to sprout out from portal spaces throughout the liver, dividing the hepatic parenchyma into several polyhedral portions of different sizes. Earlier studies have noted that end-stage cirrhosis can be the outcome of severe $C$. hepatica infection in rodents [2]. Significantly, Lammler et al. [3] pointed out that these late lesions are difficult to be explained, since at this time egg production is ceasing and acute focal lesions are no longer observed. As a matter of fact, when C. hepaticainduced lesions were followed through different intervals, from 1 to 3 months, the process of septal fibrosis was seen to increase progressively with time [1]. However, the outcome of $C$. hepatica-induced septal fibrosis is not known, since sufficiently prolonged studies have not been made on this regard.

Also, in a much more studied model of hepatic septal fibrosis, the one induced in rats by repeated intraperitoneal 
injections of whole pig-serum or its albumin fraction, only relatively short-term studies have been attempted [4-8].

Whether septal fibrosis, as seen in both experimental models just mentioned, will continuously evolve toward cirrhosis or will eventually stop and disappear, are questions of considerable interest to the study, not only for the understanding of the respective models, but for the general pathology of hepatic fibrosis.

The present investigation followed the changes of septal fibrosis in the liver of rats infected with $C$. hepatica, at several time intervals, from 3 months to over a year. While the amount of collagen considerably decreased with the extinction of parasitism, a residual septal fibrosis persisted. The reason why such fibrosis became irreversible was then investigated.

\section{Materials and methods}

\subsection{Animals}

A total of 54 healthy, outbred Wistar rats, weighing 200-500 g, of both sexes, were used. They were maintained in good housing conditions, with free access to a commercial balanced diet and water. All the animals were submitted to infection with 800 mature $C$. hepatica eggs, administered by a gastric tube. These eggs were isolated from the livers of experimentally infected mice through homogenization in saline followed by repeated washing and sedimentation. The clean isolated eggs were kept in a humidified Petri dish at 25-28 ${ }^{\circ} \mathrm{C}$ during a period of 28 days for embryonation.

Under general anesthesia and aseptic conditions, the animals were submitted to a surgical biopsy, with removal of a liver lobe at different times after inoculation, as follows: 3 months $(n=15), 6$ months $(n=15), 9$ months $(n=9)$ and 12 months $(n=15)$. At each time-point, blood was collected from the tail vein, for serum samples. At this same time, two animals were sacrificed soon after their portal vein system has been perfused with India-ink. The injection was made manually until the liver surface appeared uniformly black. India-ink was diluted (50-50) in 15\% gelatin.

Normal intact control rats were used for the obtaining of serum samples and for the studies of the portal vessels after India-ink perfusion.

\subsection{Histopathology}

Fragments of the liver were fixed for at least $48 \mathrm{~h}$ in buffered (pH 7.2) 10\% formalin. Paraffin-embedded sections were stained with hematoxylin and eosin, sirius-red for collagen, the PAS method with and without diastase digestion, Perls' method for iron, toluidine-blue for cellular metachromatic granules and orcein for elastic fibers.

\subsection{Transmission electron microscopy}

Tiny fragments of liver tissue (about $1 \mathrm{~mm}^{3}$ ) were immediately fixed by immersion into $4 \%$ glutaraldehyde in $0.2 \mathrm{M}$ cacodylate buffer, $\mathrm{pH} 7.4$, for $1 \mathrm{~h}$ at $4{ }^{\circ} \mathrm{C}$, washed in buffer and postfixed with $1 \%$ osmium tetroxide, dehydrated in graded concentrations of acetone and embedded in Poly-bed 812 (Embedding Media Polysciences, IVC). Selected ultrathin sections $(50-70 \mathrm{~nm})$ were made with a Reichert (Ultratome Supernova Leica) ultramicrotome and mounted on uncoated copper grids, contrasted with uranyl acetate and a lead citrate. Specimens were examined in a Zeiss EM-9 electron microscope, which was operated at an acceleration voltage of $50 \mathrm{kV}$.

\subsection{Immunofluorescence}

Fragments of liver tissue were immediately placed in liquid nitrogen for a few minutes and them kept frozen at $-70{ }^{\circ} \mathrm{C}$ in airtight boxes, until the moment they were sectioned in a cryostat at $-20^{\circ} \mathrm{C}$. The sections were submitted to an indirect immunofluorescence technique for the demonstration of collagen isotypes (I, III and IV), laminin and fibronectin. The specific anti-sera were polyclonal, obtained in rabbits (Institute Pasteur, France). They were used in dilution varying from 1:40 to 1:100. Details concerning their preparation and tests of specificity appeared elsewhere $[9,10]$. Secondary fluoresceinated anti-rabbit IgG was commercially obtained from Sigma, USA.

\subsection{Immunohistochemistry}

For the demonstration of factor VIII, dendritic cells and ED3, fragments of the liver were immediately embedded in Tissue-teck (OCT Compound-Miles Inc., Diagnostic Division, Elkhart, USA), frozen into liquid nitrogen, and cryopreserved in a freezer at $-70^{\circ} \mathrm{C}$ until the moment of sectioning.

Sections of $6 \mu \mathrm{m}$ were obtained in a cryostat at $-20^{\circ} \mathrm{C}$ on slides previously treated with poly-L-lisin at $10 \%$ (Sigma, St. Louis, MO, USA), fixed in dehydrated acetone, treated with PBS. For the blocking of non-specific ligations, sections were treated with $10 \%$ skimmed milk in PBS for 20 min at room temperature. Besides, blocking of endogenous avidin and biotin was performed with DAKO Biotin Blocking System (Code No. X0590, DAKO Corporation, USA).

For the demonstration of SM- $\alpha$ actin, TGF- $\beta$, TGF- $\beta-R$, desmin, vimentin and PDGF, paraffin sections of formalinfixed tissue were used. Antigen retrieval was accomplished through microwave treatment in citrate buffer at $\mathrm{pH}$ 6.0.

Sections were incubated with the primary antibodies overnight, at $4{ }^{\circ} \mathrm{C}$ in a humidified chamber. Primary antibodies were diluted in $2 \%$ BSA in PBS ( $\mathrm{pH} 7.4)$. After washing in PBS, sections were incubated in $10 \%$ skimmed milk during $20 \mathrm{~min}$ for blocking non-specific ligations. The slides were then incubated with the secondary antibody: a sheep-anti-rat IgG conjugated to peroxidase (Dako envision system-labelled polymer (Dako, USA)), in the dilution of $1: 1000$ for $30 \mathrm{~min}$ at $37^{\circ} \mathrm{C}$ in a humidified chamber. Blockade of the endogenous peroxidase was done with $0.3 \%$ $\mathrm{H}_{2} \mathrm{O}_{2}$ for $30 \mathrm{~min}$, at room temperature. The color was devel- 
oped with $0.06 \% 3,3^{\prime}$-diaminobenzidine tetrahydrochloride (DAB) (Sigma) and $0.06 \% \mathrm{H}_{2} \mathrm{O}_{2}$ plus $1 \%$ dimethylsulphoxide (Sigma). Sections were counterstained with $1 \%$ methylgreen for 2 min dehydrated and mounted with Permount. Control sections in which primary antibody was either omitted or replaced by normal rat serum, were used.

\subsection{Functional liver tests}

The serum concentrations of aminotransferases (ALT and AST), alkaline phosphatase (ALK P), albumin, bilirrubins, and gamma-glutamyl transpeptidase $(\gamma-G T)$ were determined in a Hitachi 917 automatic analyzer.

\subsubsection{Hydroxyproline measurements}

Biochemical determination of hydroxyproline concentration was made according to the colorimetric method of Bergman and Loxley [11].

\subsubsection{Serum antibodies}

The levels of serum antibodies against $C$. hepatica were determined by an enzyme-linked immunosorbant assay (ELISA), to test for detection of total Ig antibodies, by using a goat anti-rat IgG conjugated to peroxidase (Sigma). The plates were sensitized overnight with $10 \mu \mathrm{g} / \mathrm{ml} / \mathrm{per}$ well of C. hepatica-egg antigen, diluted in carbonate buffer, $\mathrm{pH}$ 9.9.
Lecture was made on a microplate reader "Molecular devicesThermomax" spectrophotometer, under wavelength $450 \mathrm{~mm}$, connected to a computer with MDS-Soft Max (Version 3.0).

\section{Results}

Multifocal parasitic lesions, containing worm debris and immature eggs were already well encapsulated by 3 months after inoculation (Fig. 1A). These lesions almost disappeared by the 6th month of infection. Only clusters of eggs packed inside scattered, dense, small fibrous scars, with or without a few calcified foci, remained by the 6th month onward, sometimes in the vicinity of fibrous bands and hepatic nodules (Fig. 1B). Three months after infection, septal fibrosis appeared throughout the liver. It was represented by thin and long fibrous septa crisscrossing the hepatic parenchyma (Fig. 1C). These thin septa connected portal spaces to portal spaces. Few septa appeared projected from the capsule of old parasitic scars, and from the external capsule of the liver. The portions of hepatic tissue circumscribed by septal fibrosis, maintained the one-cell thick normal pattern of the liver parenchyma. Six months after infection, the microscopic appearance of septal fibrosis had changed. In $\mathrm{H} \& \mathrm{E}$ preparations fibrous septa were hardly discernible, since the place occupied by fibrosis appeared as a thin empty cleft, with
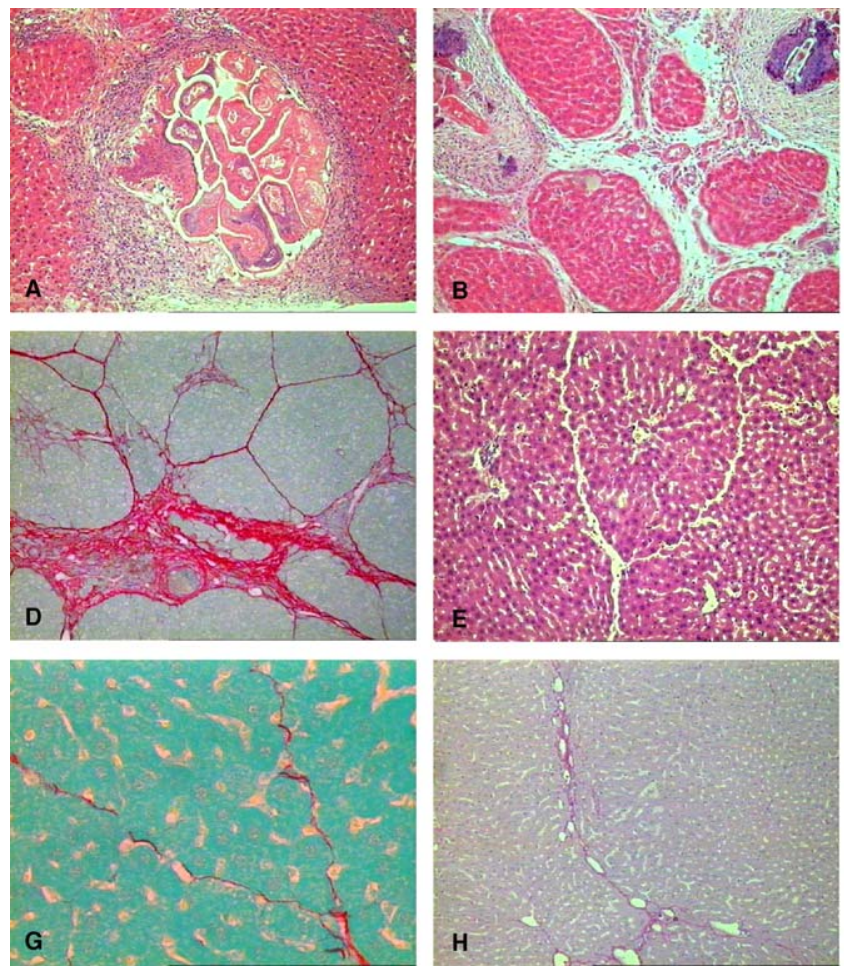
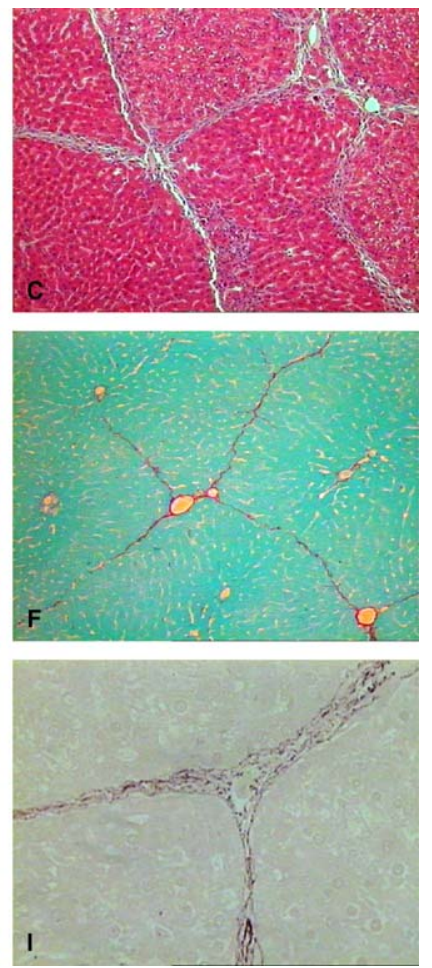

Fig. 1. (A) Dead and dying Capillaria hepatica adult worms appear packed within an encapsulated lesion present in the liver of a rat, 30 days following infection. (B) Six-month-old C. hepatica infection discloses a "cirrhotic" appearance of the liver, besides two encapsulated and calcified lesions containing worm debris and eggs. $\mathrm{H} \& \mathrm{E}, \times 100$. Pictures from $\mathrm{C}$ to I show early fibro-cellular septa $(\mathrm{C}$ and $\mathrm{D})$ contrasting with the later appearance they assume, when a few, thin, vascular, wavy and interrupted septa, containing elastic fibers, are observed (E-I). (A-C and E) Hematoxylin \& Eosin; (D and F-H) picro-sirius-red staining for collagen; (I) orcein stain for elastic tissue. Magnification $\times 100$, except $(E)$ and $(G): \times 200$. 
a few fusiform cells here and there (Fig. 1E). Picro-sirius stained slides easily disclosed that septal fibrosis was still present as delicate, thin treads of red-stained fibers The septa appeared wavy and interrupted, containing blood vessels and elastic fibers (Fig. 1F-I). These overall aspects tended to persist when materials taken from 9- and 12-month-old infections were examined. In short, besides the diminution in the amount of septal collagen from the 3rd to the 6th month following infection, all the other parameters considered, tended to remain the same, 9 and 12 months after infection. Isolation and cultivation of eggs taken from 3rd-month-old infection yielded embryonation, which did not occur when eggs from 6, 9- or 12-month-old infections were tested. Determination of hydroxyproline confirmed the impression from histological examination, showing a statistically significant difference in collagen concentration as compared to specimens taken from more prolonged infections, However, from the 6th month onward hydroxyproline measurements were about the same, indicating a stabilization of the process of collagen degradation (Fig. 2).

Collagen isotypes I and III were present within the fibrous septa at all observational stages. Collagen type IV and laminin marked basement membranes and thus disclosed the rich vascularization of the fibrous septa and also their diminution and persistence throughout the time of observation (Fig. 3A and B). Fibronectin in the fibrous septa presented decreased concentrations along the time of examination, as a matter of fact in a way comparable to what happened to the other components of the extracellular matrix evidenced by immunofluorescence.

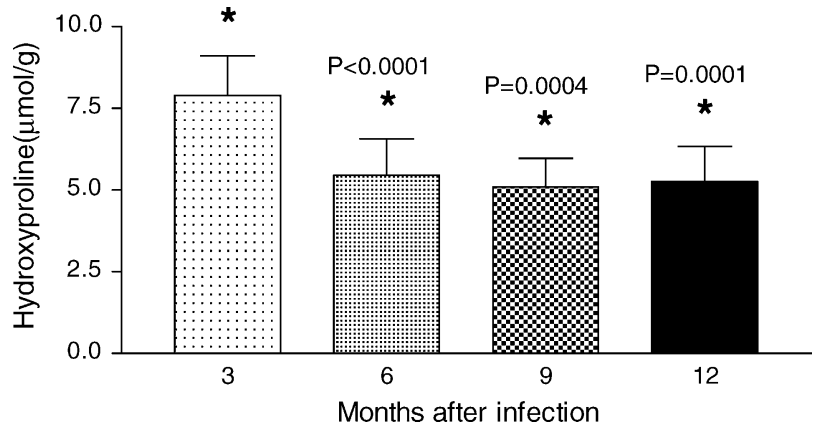

Fig. 2. Measurement of liver collagen concentration by hydroxyproline determination. Liver fragments of Wistar rats were obtained 3, 6, 9 and 12 months after infection. The hydroxyproline content per gram of liver from individual rats were determined by colorimetric assay (method of Bergman and Loxley [11]).

The cellular elements in the fibrous septa were scarce even at the 3rd month of infection, and this was even more evident during the subsequent periods. No special aspects concerning the number and distribution of the cellular components of the septa, as identified by immunohistochemistry, were apparent. Cells positive for desmin, vimentin, PDGF, TGF$\beta$, TGF- $\beta-R$, ED3 were few and appeared occasionally within the thin fibrous septa. Actin was positive for a few cells in the septa, but appeared prominent by revealing the muscular walls of blood vessels (Fig. 3C), the endothelial cells of which appeared positive for factor VIII (Fig. 3D). Late septa, from the 6th to the 12th months after inoculation, practically contained only a few collagen fibers and small thin-walled, blood vessels.
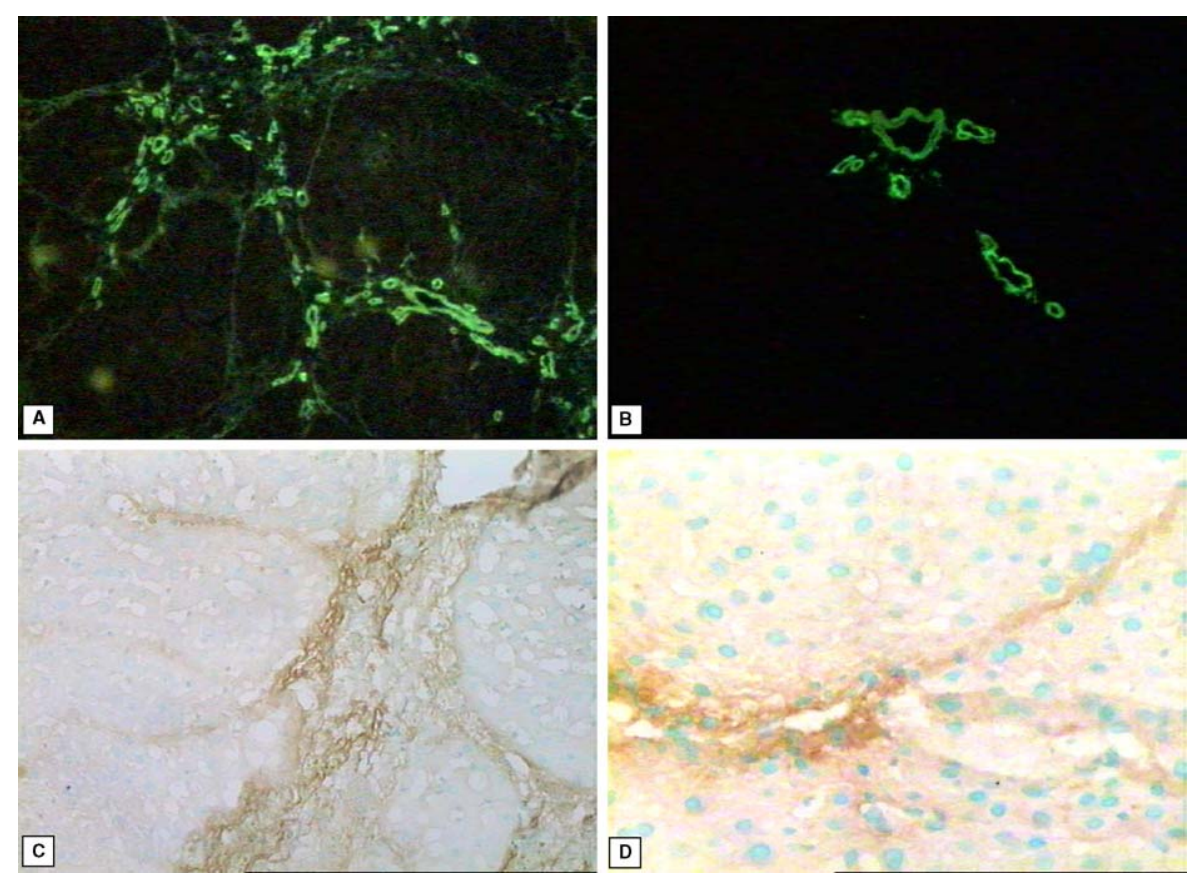

Fig. 3. (A) Laminin marks the basement membrane of blood vessels and reveals the rich presence of them in early $C$. hepatica-induced septal fibrosis of the liver and (B) their diminution at a later period of the infection ( 6 months). Indirect immunofluorescence, $\times 200$. (C) The muscular walls of blood vessels appear positively stained for actin and the endothelial cells (D) are marked for factor VIII, thus revealing the prominent presence of blood vessels within the C. hepatica-induced fibrosis in the liver of rats. Immunohistochemistry, $\times 200$. 

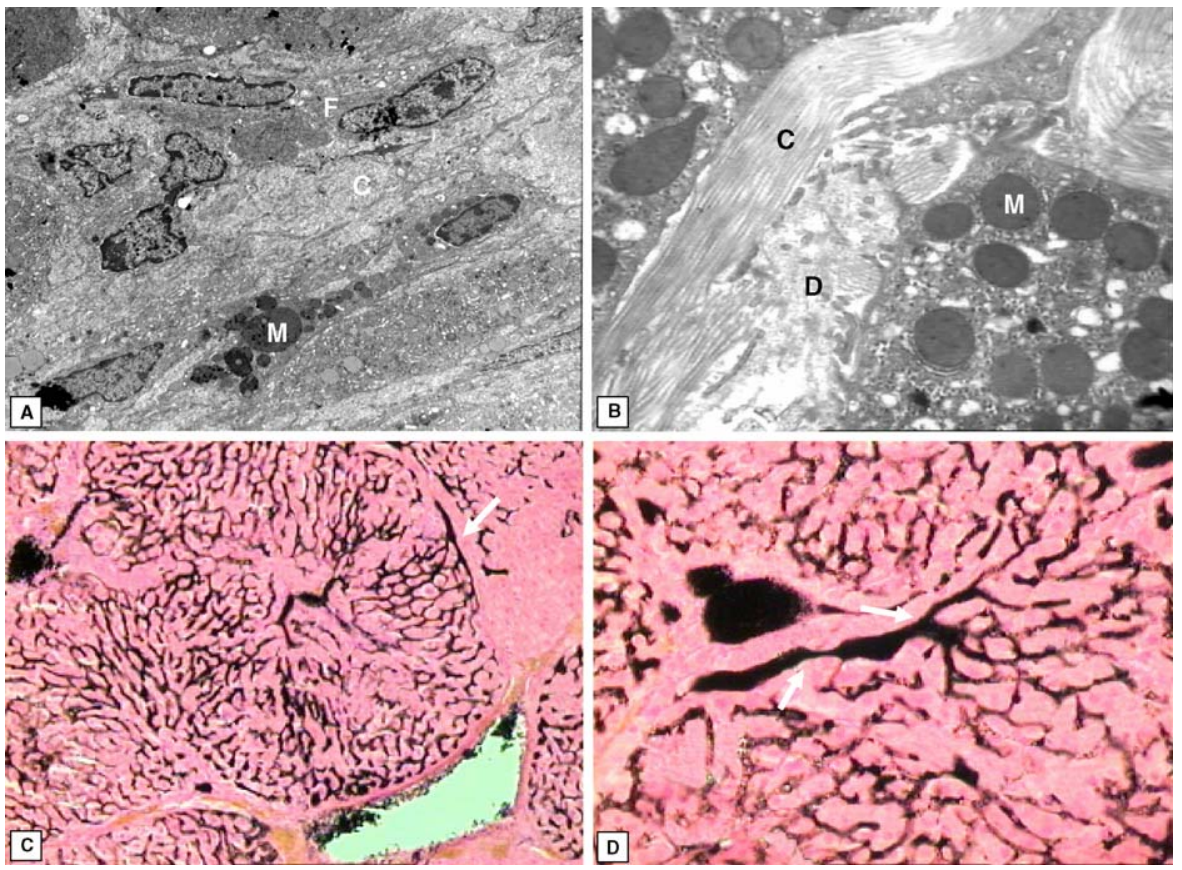

Fig. 4. (A) Ultrastructure of an early septum reveals fibroblasts and loosely arranged collagens fibers. F: fibroblast, M: mast cell, C: collagen fibrils. EM, $\times 3000$. (B) Late septum formed by densely packed collagen fibrils and no cells. C: collagen, D: Disse space, M: mitochondrium. EM, $\times 7000$. (C and D) Septal vessels filled with Indian-ink drain directly into hepatic sinusoids. (C) $\times 100$ and (D) $\times 200$.

Ultrastructurally, the septa seen by the 3rd month exhibited a mixture of several cell types, with predominance of fibroblasts and a few myofibroblasts. Other cells eventually identified in the septa included fat-storing cells (HSC), mast cells, eosinophils and lymphocytes (Fig. 4A). From the 6th month on, the septa contained densely packed collagen fibers and one or other of the cells previously mentioned (Fig. 4B).

Sections of the liver previously injected with India-ink revealed that the fibrous septa contained vessels that terminated directly into sinusoids. Some of these vessels appeared straight, thin, without collaterals, running for some distance within the septum, before dividing into several terminal ramifications and going towards the sinusoids (Fig. 4C and D). These aspects were observed in all specimens taken from infected rats. Normal controls showed that portal vessels tended to form an arcing structure near the periportal parenchymal cords before giving off short communications to the sinusoids.

The exploration of liver function failed to reveal major alterations. The data obtained in several periods were within normal limits. The only exception was a slight increase in alkaline phosphatase, of $367.7 \pm 5.0 \mathrm{IU} / 1$ (controls $139.4 \pm 43.2)$ and aminotransferases, ALT 162.2 $\pm 3.5 \mathrm{IU} / \mathrm{l}$ (controls 140.8 \pm 7.8 ) and AST 92.2 $\pm 22.4 \mathrm{IU} / 1$ (controls $68.4 \pm 14.7$ ) levels by the 3rd month of infection, a moment when focal necro-inflammatory parasitic lesions were prominent.

Levels of serum anti-C. hepatica antibodies are depicted in Fig. 5.

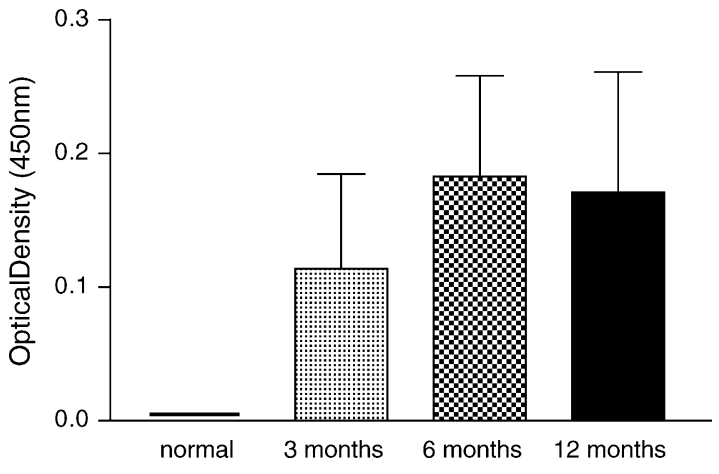

Fig. 5. Levels of antibodies detected in the serum of the rats after 3,6 and 12 months of the infection.

\section{Discussion}

The interesting fact about $C$. hepatica-induced fibrosis in rats is that its course is progressive during the first 3 months of infection [1], but it soon stops and starts revealing signs of progressive regression by the 6th month onward. This change of course correlates well with the extinction of parasitism, as demonstrated in the present study. These observations are in keeping with the well-established notion that not only hepatic fibrosis is reversible, but that the best way to produce such regression is to remove its cause [12-14]. To stay in the field of hepatic fibrosis due to parasitic diseases, a good example of this principle can be seen in both human and experimental schistosomiasis, when not only hepatic fibrosis, but its associated manifestations of portal hypertension, can com- 
pletely disappear following curative treatment $[9,10,14,15]$. However, there are hepatic fibroses with different morphology and significance. Shibayama and Nakata [15] demonstrated that hepatic fibrosis induced by pig-serum injections into rats did not have a functional expression. By measuring oxygen consumption, the velocity of blood perfusion, and other parameters, they concluded that fibrosis by itself does not disturb liver function. Lortat-Jacob et al. [17] made the interesting observation that hepatic fibrosis in experimental schistosomiasis treated with interferon presented a differential sequence of disappearance, with perisinusoidal fibrosis disappearing first and more rapidly than that of periovular granulomas. There are several morphological types of hepatic fibrosis and they do not have the same behavior as far as reversibility is concerned. There is a long controversy regarding cirrhosis reversibility, meaning that in some circumstances fibrosis may indeed be irreversible [18-20]. However, the debate is still lingering regarding human and experimental cirrhosis [21,22]. Although Wanless et al. [23] have listed a series of histological findings, collectively called the "hepatic repair complex", supposed to represent parameters of cirrhosis regression, no one has ever documented the morphologic transition of a human cirrhotic liver to a normal one. When the present study was made 6 months after $C$. hepatica infection, the data showing extinction of parasitism and decrease of septal fibrosis left us under the impression that the whole structure of the liver would subsequently return to normal. However, septal fibrosis became stabilized for the next 6 months. Fibrosis then appeared to be merely a supportive stroma of a new vascular disposition. Adaptation of the liver on face of a continuous aggression may result in fibrosis with vascular changes. During the process of excess fibrous tissue removal, that tissue serving as a supporting stroma for physiologically functioning blood vessels tends to be spared. The expectation that such septal fibrosis can be removed is similar to expecting removal of the normal portal fibrous tissue by anti-fibrotic treatment or other means. Present observations support the conclusion that associated vascular changes may be a decisive factor regarding the reversibility of fibrosis. This would also explain why cirrhosis is clinically reversible, as recently discussed by Friedman [21], although probably morphologically irreversible.

\section{References}

[1] Ferreira LA, Andrade ZA. Capillaria hepática: a cause of septal fibrosis of the liver. Mem Inst Oswaldo Cruz 1993;88:441-7.

[2] Luttermoser GW. An experimental study of Capillaria hepatica in the rat and the mouse. Am J Hyg 1938;27:321-40.
[3] Lammler G, Zahner H, Vollerthun R, Rudolph R. Egg production and host reaction in Capillaria hepatica infection of Mastomys natalensis. In: Soulsby EJL, editor. Parasitic zoonoses: clinical and experimental studies. New York: Academic Press; 1974.

[4] Andrade ZA. Contribution to the study of septal fibrosis of the liver. Int J Exp Pathol 1991;72:553-62.

[5] Ballardini G, Faccani A, Beti S, et al. Sequential behaviour of intracellular matrix glycoproteins in an experimental model of hepatic fibrosis. Virchows Arch (Cell Pathol) 1985;49:317-24.

[6] Rubin E, Hutterer F, Popper H. Experimental hepatic fibrosis without hepatocellular regeneration. A kinetic study. Am J Pathol 1968;52:111-9.

[7] Paronetto F, Popper H. Chronic liver injury induced by immunologic reactions. Cirrhosis following immunization with heterologous sera. Am J Pathol 1996;40:1087-101.

[8] Nakano M. Early morphological changes of porcine serum-induced hepatic fibrosis. Acta Path Jpn 1980;3:415-22.

[9] Andrade ZA, Grimaud JA. Evolution of the schistosomal hepatic lesions in mice after curative chemotherapy. Am J Pathol 1986;124:59-65.

[10] Andrade ZA, Grimaud JA. Morphology of chronic collagen resorption (a study on the late stages of schistosomal granuloma involution). Am J Pathol 1988;132:389-99.

[11] Bergman I, Loxley R. Two improved and simplified methods for the spectophotometric determination of hidroxyprolin. Anal Chem 1963;35:1961-5.

[12] Arthur MJP. Matrix degradation in the liver. Sem Liver Dis 1990;10:47-55.

[13] Poynard T, Mchutchinson J, Manns M, et al., for the PEG-FIBROSIS Project Group. Impact of pegylated interferon alfa-2a and ribavirin on liver fibrosis in patients with chronic hepatitis C. Gastroenterology 2002;122:1303-13.

[14] Mohamed-Ali Q, Doehring-Schwerdtfeger E, Abdel-Rahim IM, et al. Ultrasonographic investigation of periportal fibrosis in children with Schistosoma mansoni infection: reversibility of morbidity seven months after treatment with praziquantel. Am J Trop Med Hyg 1991;44:444-51.

[15] Shibayama Y, Nakata K. Significance of septal fibrosis for disturbance of hepatic circulation. Liver 1992;12:22-5.

[17] Lortat-Jacob H, Baltzer F, Desmouliere A, Peyrol S, Grimaud JA. Lobular - but not periovular - inhibition of collagen deposition in the liver of $S$. mansoni-infected mice using interferon- $\gamma$. J Hepatol 1997;26:894-903.

[18] Perez-Tamayo R. Cirrhosis of the liver: a reversible disease? In: Sommers SC, Rosen PP, editors. Pathology annual, vol. 14. New York: Appleton-Century-Crofts; 1975. p. 183-213.

[19] Perez-Tamayo R. Degradation of collagen: pathology. In: Weiss JB, Jayson MIV, editors. Collagen in health and disease. London: Churchil Livinstone; 1982. p. 135-59.

[20] Quinn OS, Higginson J. Reversible and irreversible changes in experimental cirrhosis. Am J Pathol 1965;47:353-69.

[21] Friedman SL. Liver fibrosis-from bench to bedside. J Hepatol 2003;38:38-53.

[22] Iredale JP, Benyon RC, Pickering J, et al. Mechanisms of spontaneous resolution of rat liver fibrosis: hepatic stellate cell apoptosis and reduced hepatic expression of metalloproteinase inhibitors. J Clin Invest 1998;102:538-49.

[23] Wanless IR, Nakashima E, Sherman M. Regression of human cirrhosis. Morphologic features and the genesis of incomplete septal cirrhosis. Arch Pathol Lab Med 2000;124:1599-607. 